Commentary on Maria and Andrea: Comparing Positive and Neqative Outcome Cases in an Online, Clinician-Guided, Self-Help Intervention for Panic Disorder; and

Daydreamer and Night Owl: Comparing Positive and Negative Outcome Cases in an Online, Clinician-Guided, Self-Help Intervention for Social Anxiety Disorder

\title{
Strengths and Limitations of Internet-Based Cognitive-Behavioral Treatments for Anxiety Disorders
}

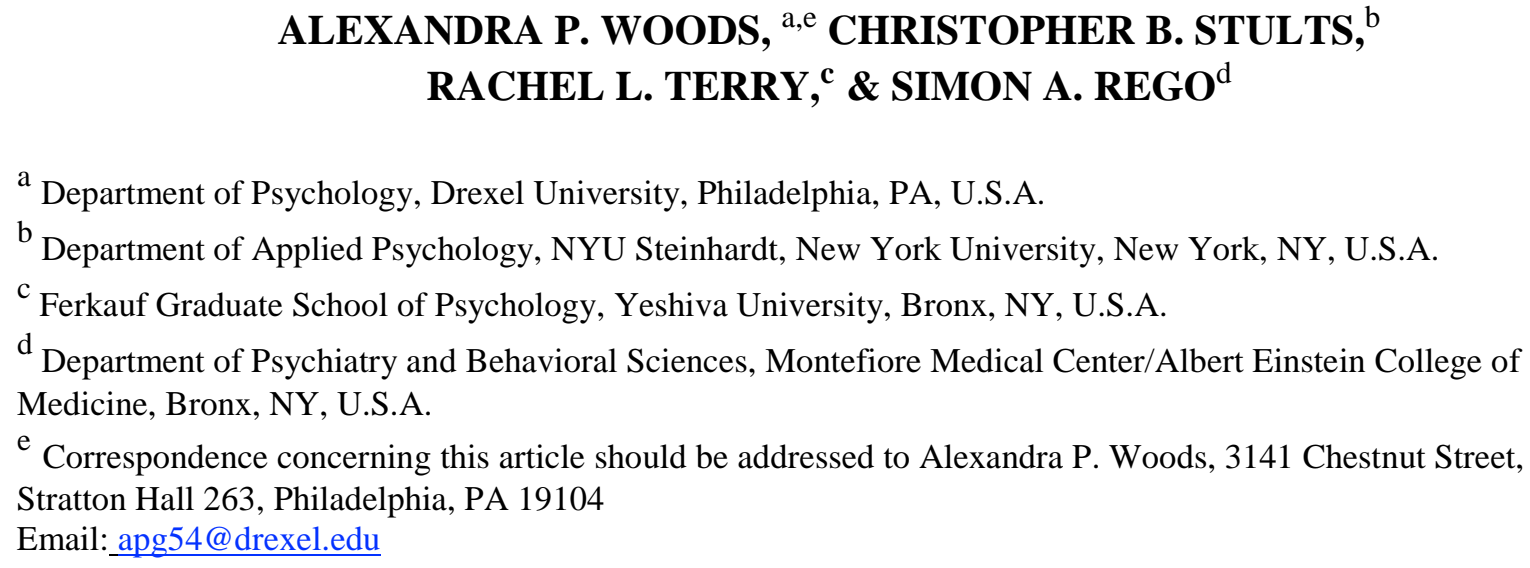

\begin{abstract}
This commentary discusses the strengths and limitations of internet-based cognitive-behavioral treatments (ICBT) for anxiety disorders, as highlighted by the case studies described in Ciuca, Berger, and Miclea (2017), and in Schulz, Vincent, and Berger (2017). In comparing the course of treatment for patients with contrasting clinical outcomes, the authors offer insights into the many benefits and challenges of ICBT. Suggestions for improving ICBT interventions for anxiety disorders, implications for implementation and dissemination, and ethical considerations are discussed.
\end{abstract}

Key words: cognitive-behavioral therapy; internet-based treatments; social anxiety disorder; panic disorder; anxiety; case studies; clinical case studies

\section{STRENGTHS AND LIMITATIONS OF INTERNET-BASED COGNITIVE- BEHAVIORAL TREATMENTS FOR ANXIETY DISORDERS}

Internet-based cognitive-behavioral therapies (ICBT) have emerged as a promising alternative or adjunct to the traditional format of CBT treatment conducted face-to-face with an experienced clinician. Ciuca, Berger, and Miclea (2017) and Schulz, Vincent, and Berger (2017) each present a pair of case studies comparing the course of treatment between positive-outcome and relatively negative-outcome patients receiving ICBT interventions for social anxiety disorder and panic disorder, respectively. Using the Individual-Case-Comparison (ICC) method 
(Fishman, 2011; Fishman, Messer, Edwards, \& Dattilio, 2017), patients with contrasting treatment outcomes were selected from the experimental condition of a randomized controlled trial and described in detail. The examination of these complex individual cases can generate possible explanations for how the process of change occurs in ICBT, as well as who is most likely to benefit from an ICBT intervention. The case studies succeed in highlighting the many benefits of delivering CBT via an online platform, as well as limitations which present opportunities for further research.

\section{STRENGTHS OF ICBT}

\section{Increased Access to Care}

One advantage of ICBT interventions such as those described in the Ciuca et al. (2017) and Schulz et al. (2017) articles is the potential for increased access to evidence-based treatments for consumers. Indeed, this need exists for a wide range of mental health disorders (Knickman, Krishnan, \& Pincus, 2016). According to data collected by the Substance Abuse and Mental Health Services Administration (SAMHSA) in 2015, nearly one in five Americans (17.9\%) met criteria for a mental health disorder in the previous year (Center for Behavioral Health Statistics and Quality, 2016). Yet less than half of those Americans (43.1\%) with mental illnesses sought treatment during the last year (Center for Behavioral Health Statistics and Quality, 2016).

Anxiety disorders are the most prevalent class of mental disorders (Kessler, Ruscio, Shear, \& Wittchen, 2009). However, while there is an extensive body of research on the efficacy of cognitive-behavioral treatments for anxiety disorders, there are still significant barriers that prevent patients from accessing these treatments (Gunter \& Whittal, 2010). In particular, avoidance and symptoms inherent in anxiety disorders may directly interfere with individuals seeking traditional psychotherapy (i.e., once-weekly, in office, face-to-face). For instance, Andrea in the Ciuca et al. (2017) article was terrified to leave her home unless accompanied by a trusted companion. Her panic symptoms were exacerbated by comorbid medical problems that limited her mobility. Night Owl, the socially anxious patient described by Schulz et al. (2017), avoided many social interactions due to his intense fear that his social anxiety would manifest in noticeable physical symptoms (e.g., blushing, red spots). Like Andrea, he may be an ideal candidate for an ICBT intervention. As demonstrated with these patients, online, clinicianguided, self-help interventions have the potential to lessen disorder-related barriers to engagement in effective mental health treatments.

The gap in mental health care utilization in the United States is potentially exacerbated by the recent implementation of the Affordable Care Act (ACA; Creedon \& Lê Cook, 2016). With the ACA, the pool of insured Americans increased by nearly 20 million people (Garrett \& Gangopadhyaya, 2016). The ACA also requires that all health insurance plans cover mental health services. Therefore, many new potential consumers of mental health services have recently entered a mental health care marketplace that is unlikely to be capable of efficiently addressing the increased demand for services. These considerations suggest that the proportion of Americans with untreated mental health problems will likely remain unchanged despite the national expansion of health insurance coverage (Beronio, 2014). 
In addition to recent changes in health insurance coverage in the United States, there are practical barriers to seeking treatment for mental health problems. These barriers may be mitigated by ICBT interventions, particularly for anxiety disorders (Olfson et al., 2000). For example, long wait lists may result from an insufficient number of providers in some geographic areas (Carroll, 2016). There can often be issues with consistent transportation to and from appointments, especially if patients must travel long distances to meet with providers in rural contexts. Parents of young children and those caring for aging parents may find it difficult to arrange for alternate caregivers to allow attendance at weekly therapy appointments. Additionally, working Americans may be unable to coordinate their work schedules with recurring mental health appointments, many of which take place during traditional business hours.

Finally, ICBT may address potential gaps in mental health care utilization by offering mental health services via online or mobile platforms that may be more acceptable to younger users who increasingly use electronic forms of communication. As detailed in the Ciuca et al. (2017) and Schulz et al. (2017) case studies, the more successful outcomes were demonstrated by the younger users (i.e., Maria and Daydreamer, respectively).

\section{Accommodation to Users}

At this time, ICBT interventions are generally not thought of as replacements for traditional psychotherapy. Rather, they are considered to be alternative options for specific patient populations (e.g., patients with significant barriers to traditional treatment) or for specific disorders with a sufficient evidence base to substantiate the use of an online-only treatment. Better Help, one of the most popular online therapy companies in the United States, specifies on its website that its services are "not intended to be a universal replacement for traditional psychotherapy” (Better Help, 2017).

However, ICBT does have the potential to provide help to users who would not otherwise initiate traditional face-to-face therapy. As detailed by Schulz et al. (2017), Daydreamer had considered seeing a therapist for years but was unable to muster the courage to make an appointment. Indeed, the stigma attached to being seen entering a therapist's office was a significant deterrent for him. Furthermore, a positive experience using online mental health platforms may encourage users to eventually engage in face-to-face therapy, as Night Owl felt prepared to do at the end of his online treatment.

\section{Public Health Implications}

ICBT interventions are also more conducive to public health efforts designed to improve the mental health of populations. In particular, ICBT interventions are more easily scaled up to reach the needs of a large number of people, as compared to individual face-to-face interventions (Fairburn \& Patel, 2017). For instance, a county’s public health department could purchase a bulk license for its residents to access an ICBT platform as an adjunct to the mental health services it already provides at its various clinics and hospitals. Multiple users could simultaneously access the ICBT intervention at their convenience, which would also be a way to reduce the demand on potentially overburdened mental health agencies. One example of this is MindSpot, a government-funded online treatment clinic for Australian adults with anxiety or 
depression (Titov et al., 2015). Participants who completed MindSpot were found to have symptom improvement comparable to face-to-face treatments in effect size and clinical change. Thus, ICBT could be an important step in improving the overall health of the population.

\section{Applicability of CBT to Internet-Based Treatment}

As demonstrated in the two sets of case studies, cognitive-behavioral therapy (CBT) may lend itself particularly well to the development of an adapted online intervention. First, CBT is generally intended to be a short-term form of treatment as compared to other psychotherapies. This time-limited model may be more suitable for adaptation to ICBT platforms. Also, unlike some other psychotherapeutic models which are intended to unfold over time via successive insights gained in-session that are unique to each patient, CBT contains general principles and techniques that can be crafted into content-driven modules. As discussed in the Ciuca et al. (2017) article, CBT-informed psychoeducation, techniques for decreasing hyperarousal, cognitive restructuring skills, exposure exercises, positive emotions training, problem-solving training, behavioral activation, cognitive restructuring exercises, and relapse prevention strategies can all be successfully integrated into online platforms.

In addition, transdiagnostic ICBT interventions have been developed for patients presenting with comorbid anxiety disorders. For example, participants who completed a 6session intervention combining materials from ICBT programs for generalized anxiety disorder, social phobia, and panic disorder achieved significantly better outcomes on both disorderspecific and general measures as compared to a waitlist control group (Titov, Andrews, Johnston, Robinson, \& Spence, 2010).

While some may criticize online interventions for delivering overly structured and overly standardized content, the use of technology may allow for great flexibility in tailoring online mental health interventions to an individual's needs. Assessment measures can be integrated into ICBT platforms to closely monitor participants' progress.

Furthermore, the electronic administration of those assessments can be programmed to automatically highlight crucial items for clinicians. For example, a clinician may flag items that reflect key outcomes for a particular patient or items that indicate elevated suicide risk. In addition, ICBT interventions can flexibly deliver material that is most relevant for an individual patient based on particular clinical presentations. In one study, Carlbring et al. (2011) used assessment, clinical judgment, and patient preference to individualize a 10-week online treatment program according to specific patient characteristics and comorbidities. Participants were prescribed 10 individually-selected sections of a 16-module online CBT program for panic disorder, social phobia, generalized anxiety disorder, and depression. Those who completed this flexible intervention showed significant improvements in anxiety and depression with a moderate effect size. Additionally, ICBT platforms may allow users to better generalize CBT principles and apply relevant techniques to their daily lives. For example, a user may open up a module about common cognitive distortions as they attempt to challenge a distorted thought during their day. 


\section{LIMITATIONS OF ICBT AND OPPORTUNITIES FOR IMPROVEMENT}

\section{The Role of the Therapeutic Relationship in ICBT}

A potential limitation of ICBT interventions is the lack of face-to-face contact with a trained therapist. When meeting in person, an experienced clinician can tailor and individualize treatment for complex cases that may present with comorbidities, motivational issues, and changing needs during the course of care. In particular, face-to-face interactions for patients with social anxiety disorder also offer an opportunity to practice and receive feedback about interpersonal skills. For instance, at the end of Night Owl's participation in the program, he wrote "I still act over-controlled and too formal in a lot of social situations. Even though I do not feel fear per se, I am still very tense, and I think I don't appear very authentic” (Schulz et al., 2017, p. 236). The patient's online therapist had only communicated with him via an asynchronous messaging system and therefore was unable to comment on Night Owl's perceived social interaction deficits. Future studies should explore the use of video conferencing to provide real-time communication and social skills training.

Given the limited role of the therapist in online interventions, the impact of the therapeutic alliance on adherence and treatment outcomes in ICBT interventions remains unclear. The therapeutic alliance in face-to-face CBT for social anxiety disorder has been shown to be associated with better treatment outcomes, while lower alliance ratings were associated with higher patient drop out (Haug et al., 2015). In contrast, studies examining the therapeutic alliance in therapist-guided online interventions have produced mixed findings. Most studies suggest that therapist contact is associated with better outcomes in internet interventions (e.g., Bergman et al., 2013; Wagner et al., 2012), while others show no association (e.g., Andersson et al., 2012; Knaevelsrud \& Maercker, 2006).

Andersson et al. (2012) outline several important considerations for evaluating the mixed findings in these studies. For instance, it is possible that alliance measures have not been adequately modified to measure therapeutic alliance in electronic interactions. In addition, patient ratings of alliance with their online therapists may have been the result of treatment rather than a sign of therapeutic alliance, and alliance ratings may fluctuate at different stages in the therapeutic process. In Ciuci et al.'s (2017) panic disorder case studies, it is interesting to note that despite the struggle of the less successful client Andrea to make gains in treatment, she reported a higher therapeutic alliance than the more successful Maria, and Andrea's Skype guidance sessions with the therapist typically lasted longer than Maria's sessions. Similarly, in Schulz et al.'s (2017) social anxiety case studies, Day Dreamer had a slightly lower rating of therapeutic alliance but better clinical outcomes than Night Owl. This highlights the fact that even if ICBT patients can develop a strong therapeutic alliance and consistent engagement with the online platform, there are some who appear to need more support to achieve symptom reduction. 


\section{Increasing Patient Motivation in Early Stages of ICBT}

Research suggests that in-person contact with a therapist may increase treatment adherence and prevent dropout. Carlbring et al. (2005) found that compared with face-to-face therapy, participants in an ICBT group were significantly less likely to finish treatment modules and tended to have lower ratings of treatment credibility. Similar results were found by Kiropoulos et al. (2008) in which ratings of treatment compliance and understanding of CBT material were higher in face-to-face therapy compared with those receiving an ICBT intervention. When comparing varied levels of therapist involvement in online interventions, participants in unguided ICBT programs for social phobia were found to be much less likely to complete the course than therapist-guided ICBT for social phobia (33\% vs. 77\%; Titov, Andrews, Choi, Schwence, \& Mahoney, 2008).

These patterns were reflected in both sets of the present case studies. In Ciuca et al.'s (2017) panic disorder intervention, it was clear that Maria "was very motivated at the beginning of therapy and even read more than recommended for the first sessions. She was curious and eager to find out more about her problems and how she could reduce them” (p. 187). In contrast, it was evident in Andrea's early sessions that there were unaddressed hesitancies that would later interfere with her progress in treatment. Andrea was not reading the recommended modules, had many questions about what was going to happen in future sessions, had concerns about the safety of exposure exercises, and was avoiding parts of the modules that frightened her. The structure and pace of the protocol may not have allowed space to adequately address Andrea's concerns and enhance motivation.

The social anxiety intervention described by Schulz et al. (2017) appeared to build in more attention to motivational issues by helping participants develop an individualized case formulation, establishing reasons to initiate change, and highlighting the importance of individual goals. However, when the therapist identified early signs of Night Owl's ambivalence about treatment, it was unclear whether the therapist directly addressed his skepticism about whether the program could be beneficial for him. Later, motivational issues were only discussed when the Night Owl chose to express concerns. Mewton, Smith, Rossouw, and Andrews (2014) suggest that patient engagement and expectations about treatment may be key factors in moderating the efficacy of ICBT for social phobia. Another study showed that patient motivation significantly predicted treatment outcomes for an online OCD intervention (Diefenbach, Wootton, Bragdon, Moshier, \& Tolin, 2015). Future ICBT programs then may well benefit from the use of a readiness assessment and intervention as an introduction or prerequisite to CBT modules.

\section{Enhancing Exposure Therapy within ICBT}

Another key way to improve treatment outcomes for ICBT interventions would be to increase the focus on exposure therapy and the ways in which patients communicate with their therapists during the exposure phase of treatment. Within the field of CBT, exposure therapy is considered to be a critical ingredient in the treatment of anxiety disorders (Abramowitz, Deacon, \& Whiteside, 2012; Olatunji, Cisler, \& Deacon, 2010). Although exposure therapy has been shown to be one of the most efficacious cognitive-behavioral interventions, there are often 
barriers that interfere with maximizing its benefits (Craske, Treanor, Conway, Zbozinek, \& Bram Virvliet, 2014). The present case studies highlighted common obstacles that arise during the delivery of exposure therapy, while also calling attention to the unique challenges involved in delivering exposure therapy through an internet-based format.

\section{Increasing Therapist Support to Patients During Internet-Based Exposure Therapy}

First, the case studies illustrated the ways in which patients often struggle to engage in the beginning of exposure therapy. It is true that research has shown that patients with anxiety disorders generally perceive exposure therapy to be an acceptable, useful, and effective form of treatment (Olatunji, Deacon, \& Abramowitz, 2009). Nonetheless, the initial experience of starting exposure therapy can be a daunting undertaking for patients due to the fact that exposures purposefully evoke temporary distress (Olatunji et al., 2009).

Within the present case studies, most of the patients struggled to begin the exposure phase of treatment. For example, in Schulz et al.’s (2017) social anxiety case studies, Night Owl requested a break when he reached the exposure therapy phase of treatment due to worries that treatment was "about to get serious." While Night Owl expressed intentions to re-engage with treatment after a brief break, he also expressed concerns that he might "take the easy way out" and avoid fully engaging in the exposure sessions. Daydreamer, who in contrast to Night Owl, demonstrated a high level of motivation and consistency throughout treatment, also temporarily disengaged with the ICBT intervention once he reached the exposure portion of treatment, stating, "Although I understand that confronting things is important, I just cannot see myself doing things I have avoided for so long. It all seems too much!” (Schulz et al., 2017, p. 230). Similarly, in Ciuca et al.’s (2017) panic disorder case studies, Andrea expressed significant resistance to starting exposure work. For example, she candidly wrote to her therapist, "You won't trick me into doing exposure modules. I'm terrified about doing exposure exercises since the second module” (Ciuca et al., 2017, p. 35).

These examples illustrate the resistance patients often feel when starting exposure therapy and call attention to the fact that conducting exposure therapy can be even more challenging without face-to-face interaction between the patient and therapist (Spence et al., 2008). With this in mind, both ICBT interventions could benefit from allocating additional treatment time to help patients prepare for and fully engage in the exposure portion of treatment. Specifically, the authors might consider proactively increasing patient-therapist communication during the protocols when patients are preparing for and engaging in exposure work. Therapists may also incorporate video or phone communication at these critical points in the treatment. Additional ways to enhance exposures within an ICBT modality could involve the use of video feedback and helping patients learn new ways to appraise social situations (Clark, 2005; Hirsch, Clark, Mathews, \& Williams, 2003).

\section{Increasing Practice Across Multiple Contexts in Internet-Based Exposure Therapy}

It is critical to ensure that patients practice exposures across multiple contexts in both of the ICBT protocols. Research by Craske and colleagues (2014) underscores the importance of 
conducting exposure therapy across multiple contexts to maximize the benefits of exposure therapy. If exposures are not practiced across multiple contexts, individuals run the risk of experiencing context renewal, a process in which individuals experience a return of fear to a phobic stimulus when they confront the stimulus in a context (either internal or external) that is different than the context in which exposure therapy was conducted (Craske et al., 2014). Thus, Craske et al. (2014) emphasize that it is important to conduct interoceptive, imaginal, and in vivo exposures in many different kinds of contexts so that new learning becomes generalized.

Given the limited number of sessions that focused on exposure in the ICBT protocols, the patients were advised to independently practice exposures across multiple contexts. Yet, as demonstrated in the case comparison of Maria and Andrea, patients can vary significantly in their motivation to engage in repeated exposures across varied contexts. While Maria was motivated to continue exposure-related activities on her own and experienced benefits from her continued practice, Andrea's engagement in exposures was much more limited. Specifically, Andrea focused on exposures for two weeks and struggled to complete exposures outside of her home before the end of treatment. These behavioral differences between Maria and Andrea, as well as the differences in their treatment outcomes, suggest that brief internet-based exposure therapy may require more structure and support for exposures across multiple contexts.

\section{Addressing Safety Behaviors within Internet-Based Exposure Therapy}

We also believe that both ICBT interventions would benefit from reducing safety behaviors during exposure therapy. Research suggests that safety behaviors can lessen long-term treatment gains in exposure therapy (Barlow, 2002; Craske, Treanor, Conway, Zbozinek, \& Bram Virvliet, 2014). A recent study by van Uijen, Leer, \& Engelhard (2017) suggests that safety behaviors may be involved in relapse after exposure-based therapy for anxiety disorders. Therefore, it is important to help patients develop an awareness of safety behaviors and ultimately phase out such behaviors. In the present case studies, Night Owl and Andrea - both patients with negative outcomes-demonstrated difficulties with reducing safety behaviors while engaging in exposures. In the case of Night Owl, he completed many exposures on his own but continued to use safety behaviors, such as excessive preparation prior to social interactions. Andrea struggled to complete exposures, and when she did she complete an exposure, she typically employed safety behaviors, such as always having a friend present during the activity.

It can understandably be challenging for therapists to know whether patients are engaging in safety behaviors within an ICBT format. Without in-person interactions or direct therapist observation of exposures, many patient safety behaviors can go undetected. Potential strategies to address safety behaviors within an ICBT format may be to include a module that focuses on helping patients become more aware of their safety behaviors within feared situations. Additionally, patients can record exposures for later review by a therapist or use live videoconferencing as a way to allow for therapist assessment of safety behaviors.

\section{Ethical \& Safety Concerns}

The case studies also raise key ethical and safety concerns related to ICBT for anxiety disorders and the larger field of internet-based psychotherapy. At present, internet-based 
psychotherapeutic interventions are growing at a rapid pace and much research is needed to determine how to deliver these interventions in an effective, safe, and ethically responsible manner (Dever Fitzgerald, Hunter, Hadjistavropoulos, \& Koocher, 2010).

Currently, there is limited research on the factors that moderate the efficacy of ICBT for anxiety disorders and a lack of understanding about the contraindications for ICBT among individuals with anxiety disorders (Mewton et al., 2014). Additionally, there is a shortage of research on the negative outcomes related to internet-based psychological interventions (Andersson \& Titov, 2014). Due to the scarcity of research in these areas, case studies such as the ones reviewed in this commentary can help to generate hypotheses about potential contraindications and factors that might lead to negative outcomes. Moreover, larger research studies are needed to help us understand who might benefit and who might experience harm as a result of ICBT interventions.

Furthermore, additional research is needed to better understand how to effectively address comorbid disorders within ICBT interventions. Indeed, the case studies highlighted the complexity of treating comorbid disorders within the context of disorder-specific ICBT programs. All of the individuals featured in the case studies had comorbid disorders, and many of these individuals required alterations to their treatment protocols to address difficulties related to a comorbid disorder. For example, Maria required changes to the typical ICBT protocol for panic disorder in order to address depressive symptoms that were interfering with her progress in treatment. Ciuca et al. (2017) reported that they made the decision to address Maria's depression and found that this was a beneficial treatment decision that most likely helped her achieve later success in the treatment for panic disorder. Ideally, the field will work to develop more transdiagnostic (e.g., Barlow \& Kennedy, 2016; Ehrenreich-May \& Chu, 2013) and flexible online interventions for the many individuals who present with multiple mental health disorders.

Finally, ICBT interventions raise an ethical question about our clinical obligations to help users who abruptly stop engaging with the online programs. To what extent are practitioners obligated to follow up with ICBT patients if users no longer log-on? What are the clinical and ethical obligations if a patient only engages in the ICBT program in a sporadic manner, thereby preventing the patient from obtaining a "full dose" of treatment? How should ICBT practitioners proceed if they believe that a patient requires a higher level of care or additional support services beyond the ICBT intervention? How can online therapists intervene if they become aware of suicidal or homicidal ideation? The field would benefit from guidelines to help ICBT providers deliver treatment in a way that is informed by ethical principles and clinical best practices.

\section{CONCLUSION}

In today's world, the development of new technology is outpacing our attempts to test the implications and safety of its clinical uses. Case studies such as those discussed here are therefore critically important in evaluating the efficacy, dissemination, and implementation of ICBT for anxiety disorders. Electronically-delivered interventions offer great advantages, including increased access to treatment, a potential bridge to in-person therapy, and opportunities for large-scale delivery. We also suggest several ways in which ICBT interventions for anxiety may be improved, such as increased attention to patient motivation at the onset of treatment and 
specific strategies to enhance exposures, which we view as a critical ingredient to the treatment of anxiety disorders. Given the current state of the research literature on internet-based treatment interventions, we conclude that the efficacy of ICBT appears promising but has not yet been established. While there is currently little focus on internet-delivered interventions in clinical psychology doctoral training programs, we recommend that the next generation of researchers and clinicians be encouraged to further evaluate internet-based treatments, consider their limitations, and explore the ethical questions that arise in this new treatment format.

\section{REFERENCES}

Abramowitz, J. S., Deacon, B. J., \& Whiteside, S. P. (2012). Exposure therapy for anxiety: Principles and practice. New York: Guildford Press.

Andersson, G., Paxling, B., Wiwe, M., Vernmark, K., Felix, C. B., Lundborg, L., ... \& Carlbring, P. (2012). Therapeutic alliance in guided internet-delivered cognitive behavioural treatment of depression, generalized anxiety disorder and social anxiety disorder. Behaviour Research and Therapy, 50(9), 544-550.

Andersson, G., \& Titov, N. (2014). Advantages and limitations of Internet-based interventions for common mental disorders. World Psychiatry, 13(1), 4-11.

Barlow, D.H., \& Kennedy, K.A. (2016). New approaches to diagnosis and treatment in anxiety and related emotional disorders: A focus on temperament. Canadian Psychology/Psychologie canadienne, 57, 8-20.

Bergman Nordgren, L., Carlbring, P., Linna, E., \& Andersson, G. (2013). Role of the working alliance on treatment outcome in tailored internet-based cognitive behavioural therapy for anxiety disorders: randomized controlled pilot trial. JMIR Research Protocols, 2(1), e4.

Beronio, K. (2014). Affordable Care Act will expand mental health and substance use disorder benefits and parity protections for 62 million Americans. Mental Health, 2.

Better Help (2017). Frequently Asked Questions. Retrieved from https://www.betterhelp.com/faq/

Carlbring, P., Maurin, L., Törngren, C., Linna, E., Eriksson, T., Sparthan, E., ... \& Andersson, G. (2011). Individually-tailored, Internet-based treatment for anxiety disorders: A randomized controlled trial. Behaviour Research and Therapy, 49(1), 18-24.

Carlbring, P., Nilsson-Ihrfelt, E., Waara, J., Kollenstam, C., Buhrman, M., Kaldo, V., ... \& Andersson, G. (2005). Treatment of panic disorder: live therapy vs. self-help via the Internet. Behaviour Research and Therapy, 43(10), 1321-1333.

Carroll, A. E. (2016, November 7, 2016). A doctor shortage? Let's take a closer look. The New York Times. Retrieved from https://www.nytimes.com/2016/11/08/upshot/a-doctorshortage-lets-take-a-closer-look.html

Center for Behavioral Health Statistics and Quality (2016). Key substance use and mental health indicators in the United States: results from the 2015 National Survey on Drug Use and Health. Substance Abuse and Mental Health Services Administration. https://www.samhsa.gov/data/sites/default/files/NSDUH-FFR1-2015/NSDUH-FFR12015/NSDUH-FFR1-2015.pdf.

Ciuca, A.M., Berger, T., \& Miclea. M. (2017). Maria and Andrea: Comparing positive and negative outcome cases in an online, clinician-guided, self-help intervention for panic disorder. Pragmatic Case Studies in Psychotherapy, 13(3), Article 1, 173-216. Available: http://pcsp.libraries.rutgers.edu 
Clark, D. M. (2005). The essential handbook of social anxiety for clinicians. New York, NY: John Wiley \& Sons Ltd.

Craske, M. G., Treanor, M., Conway, C. C., Zbozinek, T., \& Vervliet, B. (2014). Maximizing exposure therapy: an inhibitory learning approach. Behaviour Research and Therapy, 58, 10-23.

Creedon, T. B., \& Lê Cook, B. (2016). Access to mental health care increased but not for substance use, while disparities remain. Health Affairs, 35(6), 1017-1021.

Deacon, B. J., \& Abramowitz, J. S. (2004). Cognitive and behavioral treatments for anxiety disorders: A review of meta-analytic findings. Journal of Clinical Psychology, 60(4), 429-441.

Deacon, B., Kemp, J. J., Dixon, L. J., Sy, J. T., Farrell, N. R., \& Zhang, A. R. (2013). Maximizing the efficacy of interoceptive exposure by optimizing inhibitory learning: A randomized controlled trial. Behaviour Research and Therapy, 51(9), 588-596.

Dever Fitzgerald, T., Hunter, P. V., Hadjistavropoulos, T., \& Koocher, G. P. (2010). Ethical and legal considerations for internet-based psychotherapy. Cognitive Behaviour Therapy, 39(3), 173-187.

Diefenbach, G. J., Wootton, B. M., Bragdon, L. B., Moshier, S. J., \& Tolin, D. F. (2015). Treatment Outcome and Predictors of Internet Guided Self-Help for ObsessiveCompulsive Disorder. Behavior Therapy, 46(6), 764-774.

Ehrenreich-May, J., \& Chu, B.C. (2013). Transdiagnostic treatments for children and adolescents: Principles and practice. New York: Guilford.

Fairburn, C. G., \& Patel, V. (2014). The global dissemination of psychological treatments: a road map for research and practice. American Journal of Psychiatry, 171(5), 495-498.

Fishman, D. B. (2011). The "Individual-Case-Comparison" Method for Systematically Comparing Good-Outcome and Poor-Outcome RCT Clients: Editor's Introduction. Pragmatic Case Studies in Psychotherapy, 7(2), Article 1, 242-245. Available: http://pcsp.libraries.rutgers.edu

Fishman, D.B., Messer, S.B., Edwards, D.J.A., \& Dattilio, F.M. (2017). Case studies within psychotherapy trials: Integrating qualitatve and quantitative methods. New York: Oxford.

Garrett, A. B., \& Gangopadhyaya, A. (2016). Who gained health insurance under the ACA, and where do they live? ACA Implementation - Monitoring and Tracking. Urban Instritute. Retrieved from http://www.urban.org/sites/default/files/publication/86761/2001041-whogained-health-insurance-coverage-under-the-aca-and-where-do-they-live.pdf

Gunter, R. W., \& Whittal, M. L. (2010). Dissemination of cognitive-behavioral treatments for anxiety disorders: Overcoming barriers and improving patient access. Clinical Psychology Review, 30(2), 194-202.

Haug, T., Nordgreen, T., Öst, L. G., Tangen, T., Kvale, G., Hovland, O. J., ... \& Havik, O. E. (2015). Working alliance and competence as predictors of outcome in cognitive behavioral therapy for social anxiety and panic disorder in adults. Behaviour Research and Therapy, 77, 40-51.

Heimberg, R.G., Dodge, C.S., Hope, D.A., Kennedy, C.R., Zollo, L.J., \& Becker, R.E. (1990). Cognitive behavioral group treatment for social phobia: Comparison with a credible placebo control. Cognitive Therapy and Research, 14, 1-23.

Hirsch, C. R., Clark, D. M., Mathews, A., \& Williams, R. (2003). Self-images play a causal role in social phobia. Behaviour Research and Therapy, 41(8), 909-921. 
Kessler, R. C., Ruscio, A. M., Shear, K., \& Wittchen, H.-U. (2009). Epidemiology of anxiety disorders Behavioral Neurobiology of Anxiety and its Treatment (pp. 21-35): Springer.

Kiropoulos, L. A., Klein, B., Austin, D. W., Gilson, K., Pier, C., Mitchell, J., \& Ciechomski, L. (2008). Is internet-based CBT for panic disorder and agoraphobia as effective as face-toface CBT?. Journal of Anxiety Disorders, 22(8), 1273-1284.

Knaevelsrud, C., \& Maercker, A. (2006). Does the quality of the working alliance predict treatment outcome in online psychotherapy for traumatized patients?. Journal of Medical Internet Research, 8(4), e31.

Knickman, J., Krishnan, R., \& Pincus, H. (2016). Improving access to effecitve care for people with people mental health and substance use. JAMA, 316(16), 1647-1648.

Mewton, L., Smith, J., Rossouw, P., \& Andrews, G. (2014). Current perspectives on Internetdelivered cognitive behavioral therapy for adults with anxiety and related disorders. Psychology Research and Behavior Management, 7, 37-46.

Olatunji, B. O., Cisler, J. M., \& Deacon, B. J. (2010). Efficacy of cognitive behavioral therapy for anxiety disorders: a review of meta-analytic findings. The Psychiatric Clinics of North America, 33(3), 557-577.

Olatunji, B. O., Deacon, B. J., \& Abramowitz, J. S. (2009). The Cruelest Cure? Ethical Issues in the Implementation of Exposure-Based Treatments. Cognitive and Behavioral Practice, 16(2), 172-180.

Olfson, M., Guardino, M., Struening, E., Schneier, F. R., Hellman, F., \& Klein, D. F. (2000). Barriers to the treatment of social anxiety. American Journal of Psychiatry, 157(4), 521527.

Rapee, R. M., \& Heimberg, R. G. (1997). A cognitive-behavioral model of anxiety in social phobia. Behaviour Research and Therapy, 35(8), 741-756.

Rozental, A., Andersson, G., Boettcher, J., Ebert, D. D., Cuijpers, P., Knaevelsrud, C., ... \& Carlbring, P. (2014). Consensus statement on defining and measuring negative effects of Internet interventions. Internet Interventions, 1(1), 12-19.

Schulz, A., Vincent, A., \& Berger, T. (2017). Daydreamer and Night Owl: Comparing positive and negative outcome cases in an online, clinician-guided, self-help intervention for social anxiety disorder. Pragmatic Case Studies in Psychotherapy, 13(3), Article 2, 217252. Available: http://pcsp.libraries.rutgers.edu

Spence, S. H., Donovan, C. L., March, S., Gamble, A., Anderson, R., Prosser, S., . . Kenardy, J. (2008). Online CBT in the Treatment of Child and Adolescent Anxiety Disorders: Issues in the Development of BRAVE-ONLINE and Two Case Illustrations. Behavior and Cognitive Therapy, 36(4), 411-430. doi: 10.1017/S135246580800444X

Titov, N., Andrews, G., Choi, I., Schwencke, G., \& Mahoney, A. (2008). Shyness 3: randomized controlled trial of guided versus unguided Internet-based CBT for social phobia. Australian \& New Zealand Journal of Psychiatry, 42(12), 1030-1040.

Titov, N., Andrews, G., Johnston, L., Robinson, E., \& Spence, J. (2010). Transdiagnostic Internet treatment for anxiety disorders: A randomized controlled trial. Behaviour Research and Therapy, 48(9), 890-899.

Titov, N., Dear, B. F., Staples, L. G., Bennett-Levy, J., Klein, B., Rapee, R. M., ... \& Purtell, C. (2015). MindSpot clinic: an accessible, efficient, and effective online treatment service for anxiety and depression. Psychiatric Services, 66(10), 1043-1050. 
van Uijen, S. L., Leer, A., \& Engelhard, I. M. (2017). Safety Behavior After Extinction Triggers a Return of Threat Expectancy. Behavior Therapy. Advance online publication. https://doi.org/10.1016/j.beth.2017.08.005

Wagner, B., Brand, J., Schulz, W., \& Knaevelsrud, C. (2012). Online Working Alliance Predicts Treatment Outcome for Posttraumatic Stress Symptoms in Arab War-Traumatized Patients. Depression and Anxiety, 29(7), 646-651. 